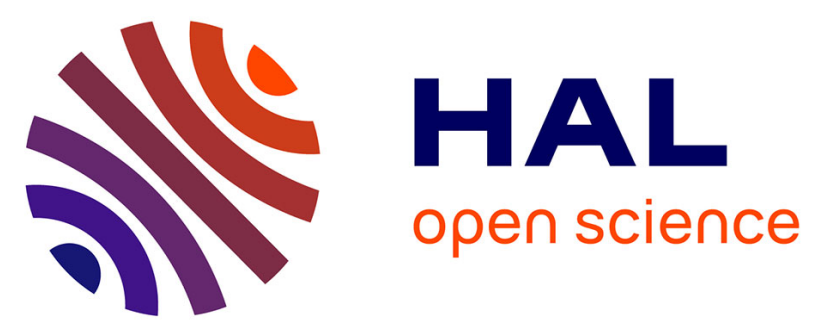

\title{
Experimental characterization of the moment-angle curve during level and slope locomotion of transtibial amputee: Which parameters can be extracted to quantify the adaptations of microprocessor prosthetic ankle?
}

Julie Davot, Marie Thomas-Pohl, Coralie Villa, Xavier Bonnet, Eric Lapeyre, Jospeh Bascou, Hélène Pillet

\section{- To cite this version:}

Julie Davot, Marie Thomas-Pohl, Coralie Villa, Xavier Bonnet, Eric Lapeyre, et al.. Experimental characterization of the moment-angle curve during level and slope locomotion of transtibial amputee: Which parameters can be extracted to quantify the adaptations of microprocessor prosthetic ankle?. Proceedings of the Institution of Mechanical Engineers. Part H, Journal of engineering in medicine, 2021, 235 (7), pp.762-769. 10.1177/09544119211006523 . hal-03349370

\author{
HAL Id: hal-03349370 \\ https://hal.science/hal-03349370
}

Submitted on 20 Sep 2021

HAL is a multi-disciplinary open access archive for the deposit and dissemination of scientific research documents, whether they are published or not. The documents may come from teaching and research institutions in France or abroad, or from public or private research centers.
L'archive ouverte pluridisciplinaire HAL, est destinée au dépôt et à la diffusion de documents scientifiques de niveau recherche, publiés ou non, émanant des établissements d'enseignement et de recherche français ou étrangers, des laboratoires publics ou privés. 


\title{
Experimental characterization of the moment-angle curve during level and slope locomotion of transtibial amputee: Which parameters can be extracted to quantify the adaptations of microprocessor prosthetic ankle?
}

\author{
Julie Davot' , Marie Thomas-Pohl'2, Coralie Villa',3, Xavier Bonnet', \\ Eric Lapeyre', Jospeh Bascou' ${ }^{1,3}$ and Hélène Pillet'
}

DOI: $10.1177 / 09544119211006523$

\begin{abstract}
In case of transtibial amputation, the deficit resulting from the loss of the lower limb can be partly compensated with a prosthetic foot and adapted rehabilitation. New prosthetic feet have been developed for transtibial amputees to mimic ankle adaptability to varying terrain. Among them, Microprocessor Prosthetic Ankles (MPA) have a microprocessor to control an electric or a hydraulic actuator to adapt ankle kinematics in stairs and slopes. The objective is to investigate parameters extracted from the moment-angle curve (MAC) and use them to compare 3 MPA during level and slope locomotion against energy storing and return (ESR) foot. Five persons with lower limb transtibial amputation successively fitted with 3 MPA (Propriofoot ${ }^{\mathrm{TM}}$, Elan ${ }^{\mathrm{TM}}$, Meridium ${ }^{\mathrm{TM}}$ ) compared to their ESR foot. The participants had 2 weeks of adaptation before data acquisition and then a 3 week wash-out period. Range of motion, equilibrium point, hysteresis, late stance energy released, and quasi-stiffness were computed on level ground and $12 \%$ slope (upward and downward) thanks to the MAC at the ankle. The study shows the relevance of MAC parameters to evaluate the behavior of MPA. In particular, compared to ESR, all MPA tested in the present study demonstrated a better angle adaptation between walking conditions but a decrease of available energy for the propulsion. Among MPA, main results were: (i) for the Propriofoot ${ }^{\mathrm{TM}}$ : an adaptation of the ankle angle without modification of the pattern of the MAC (ii) for the Elan ${ }^{\mathrm{TM}}$ : a limited adaptation of the range of motion but a modification of the energy released (iii) for the Meridium ${ }^{\mathrm{TM}}$, the highest adaptation of the range of motion but the lowest available energy of propulsion. One of the main findings of the research is to show and quantify the relationship between range of motion and energy available when using different prosthetic feet in different walking conditions.
\end{abstract}

\section{Keywords}

Walking, microprocessor-controlled feet, energy, amputation, adaptability, ramp

\section{Introduction}

Even if recent studies have shown that the gait of people with transtibial amputation can be as energetically efficient as the one of able bodied when considering young and active people, ${ }^{1,2}$ some limitations are still reported by amputee people particularly when walking in slopes. Indeed, for unimpaired people, the adaptation to slope locomotion is mainly performed by the ankle. ${ }^{3}$ Due to limited adaptability of the prosthetic ankle in stance, people with a trans-tibial amputation may adapt to slope with the residual and contralateral
'Institut de Biomécanique Humaine Georges Charpak, Arts et Métiers ParisTech, Paris, France

${ }^{2}$ Service de médecine physique et de réadaptation, Hôpital d'Instruction des Armées Percy, Clamart, France

${ }^{3} \mathrm{INI}$, Centre d'étude et de recherche sur l'appareillage des handicapés (CERAH), Créteil, France

\section{Corresponding author:}

Julie Davot, Institut de Biomécanique Humaine Georges Charpak, Arts et Métiers ParisTech, I5I boulevard de l'hôpital, Paris 75013, France.

Email: juliedavot6@gmail.com 
joints. In particular, the analysis of joint powers highlighted the role of the ipsilateral hip in slope ascent and of the residual knee in slope descent. ${ }^{4-7}$

Nowadays, new prosthetic feet have been developed for transtibial amputees in the aim to mimic ankle adaptability to varying terrain. Among them, Microprocessor Prosthetic Ankles (MPA) have a microprocessor to control an electric or a hydraulic actuator to adapt ankle kinematics in stairs and slopes. Different studies have already analyzed the improvement induced by these prosthetic feet compared to energy storing and return (ESR) feet by quantifying some parameters individually. The energetic cost decreased with the Propriofoot ${ }^{\mathrm{TM}}{ }^{8}$ compared to ESR during walking on level ground. Kinematic data have also been used to quantify the differences between prosthetic feet. The dorsiflexion in slope ascent and the plantarflexion in slope descent were closer to the ones observed during unimpaired people gait with the Propriofoot $^{\mathrm{TM}}{ }^{7}$ and the Elan ${ }^{\mathrm{TM}}{ }^{9}{ }^{9}$ However, few studies compared more than 1 MPA prosthetic foot tested by the same participant, due to the heaviness of such protocol. In static conditions, Ernst et al. investigated a cohort of people with an amputation using five different feet while standing on flat and inclined surfaces (Meridium $^{\mathrm{TM}}$, Propriofoot ${ }^{\mathrm{TM}}$, Elan ${ }^{\mathrm{TM}}$, Triton, ${ }^{\mathrm{TM}}$ and Raize $^{\mathrm{TM}}$ ) and showed that the increased range of motion and the dorsiflexion stop allow an adaptation closer to the one of asymptomatic subjects. ${ }^{10}$ In dynamic conditions, Ko et al. ${ }^{11}$ compared two different MPAs (Elan ${ }^{\mathrm{TM}}$ and Propriofoot ${ }^{\mathrm{TM}}$ ) with an ESR foot on one transtibial patient. They observed an increase of the stance phase duration during ambulation combined with a greater dorsiflexion while using the MPAs on slope. Schmalz et al. studied the effect of abrupt change of ramp inclination in patients while walking with the Meridium $^{\mathrm{TM}}$ and observed that the ankle dorsiflexion and the hip flexion patterns were similar to the ones observed in

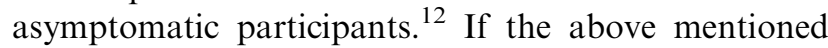
studies allowed to highlight the benefit of MPA on separated gait parameters, none have adressed the functional outcome resulting from the effect of MPA from simultaneous evaluation of these parameters.

To overcome this limitation and compare different prosthetic feet, some authors have also proposed to analyze the moment-angle curve (MAC) at the ankle in the sagittal plane during walking. ${ }^{13,14}$ Different parameters can be extracted from this curve that have been shown to be characteristics of the mechanical behavior of the prosthetic foot. First, Hansen et al. ${ }^{3}$ computed the hysteresis which is the net external energy lost in the ankle. Second, the stiffness can also be assessed. ${ }^{14,15}$ The slope of the best linear fit of the MAC characterizes the ankle behavior in the propulsion period of the stance. ${ }^{16}$ However, to the author knowledge, these parameters have never been used yet to characterize MPA behavior.

Whereas all the MPAs claims a better slope adaptation, the results in the literature are not consistent and the design parameters difficult to extract from these biomechanical studies.

Hansen and Starker ${ }^{17}$ proposed a list of five mechanical parameters of the foot determinant of the outcome of walking of persons with amputations on level ground three out of five of these parameters can be quantified from the moment-angle curve, that is therefore a way to quantify the intrinsic foot adaptation on slope and can be used to compare prosthetic feet.

The hypothesis of the present study is that parameters extracted from moment-angle curve are relevant to compare MPA adaptations while walking in slope and can be used for the design of such feet.

To test this hypothesis, the study aims at

- computing different parameters extracted from the moment-angle curve and investigating their meaning relative to the foot adaptation.

- quantifying these parameters during level and slope locomotion of 5 persons with lower limb transtibial amputation successively fitted with three microprocessor prosthetic feet compared to their usual ESR foot.

\section{Material and methods}

This study was approved by the local ethics committee (2014-A01938-39 CPP Ile de France VI Groupe Hospitalier Pitié-Salpêtrière).

\section{Participants and protocol}

Five persons with transtibial amputation were included (individual characteristics are given in Table 1) (For the participant TT01, only 2 MPA feet have been tested (Propriofoot \& Meridium).

The inclusion criteria were the following: being over 18 years old, amputated for more than 6 months, daily user of their habitual foot (HF), correctly fitted, without walking aid, for example, cane or crutches.

Three microprocessor prosthetic feet (described in the Table 2) and the habitual foot (ESR) of each individual were tested in a randomized order.

For each foot and after socket and alignment validation by one expert prosthetist (always the same), the subjects had 2 weeks to get used to the new prosthetic foot. Instrumental and functional evaluations were then performed. Between the sessions, a 3 weeks wash-out period with the habitual foot was observed before testing the next MPA foot.

\section{Motion analysis}

For quantitative analysis of gait, the subject was equipped with 54 reflective markers used to define 13 rigid segments. ${ }^{14}$ As concerns joint centers definition, the ankle joint was assessed as the middle of the malleoli for the sound limb. For the prosthetic ankle, this center was assessed as the middle of the mechanical 
Table I. Population included in the study.

\begin{tabular}{|c|c|c|c|c|c|}
\hline & TTOI & TT02 & TT03 & TT04 & TT05 \\
\hline Age (years) & 29 & 64 & 29 & 29 & 35 \\
\hline Sex & $M$ & $\mathrm{~F}$ & $M$ & $M$ & $M$ \\
\hline Amputated side & $\mathrm{R}$ & $\mathrm{L}$ & $\mathrm{R}$ & $\mathrm{L}$ & $\mathrm{L}$ \\
\hline Height $(\mathrm{cm})$ & 176 & 172 & 168 & 180 & 183 \\
\hline Mass with the prosthetic $(\mathrm{kg})$ & 82 & 68 & 70 & 66 & 95 \\
\hline Time since amputation (months) & 57 & 35 & 25 & 19 & 76 \\
\hline Habitual foot & $\begin{array}{l}\text { Sierra } \\
\text { (freedom } \\
\text { innovation) }\end{array}$ & Panthera ${ }^{\circledR}$ (Medi) & Panthera ${ }^{\circledR}$ (Medi) & Variflex $^{\circledR}$ (Ossur) & Variflex ${ }^{\circledR}$ (Ossur) \\
\hline
\end{tabular}

M: male; F: female; R: right; L: left.

Table 2. Description of the microprocessor prosthetic feet used and their technological principles.

\begin{tabular}{llll}
\hline Foot name & Manufacturer & Amplitude claimed & Comment \\
\hline Propriofoot ${ }^{\mathrm{TM}}$ & Ossur $^{\circledR}$ & $29^{\circ}$ with $18^{\circ} \mathrm{DF}$ & $\begin{array}{l}\text { Motor to modify ankle position } \\
\text { during the swing phase } \\
\text { Can adapt the hydraulic } \\
\text { response and the angle of the } \\
\text { deridium }\end{array}$ \\
& Ottobock $^{\text {TM }}$ & $\begin{array}{l}\text { Hydraulic system allowing } 14^{\circ} \\
\text { DF and } 22^{\circ} \mathrm{PF}\end{array}$ & $\begin{array}{l}\text { Can adapt the hydraulic } \\
\text { response independently in DF } \\
\text { and PF, fixed range of ankle } \\
\text { motan }\end{array}$ \\
& Endolite $^{\text {TM }}$ & Hydraulic block allowing $9^{\circ}$ with & motion
\end{tabular}

DF: dorsiflexion; PF: plantarflexion.

foot rotation axis which could be identified for the 3 MPA. For the HF, the center was taken as the symmetric of the sound side. For each patient and session, the same experimenter systematically placed the markers. Pictures of markers positions were taken in order to enhance the repeatability of positioning.

Three dimensional positions of these markers were recorded using an optoelectronic system with eight cameras (Vicon 8i, $100 \mathrm{~Hz}$, Oxford Metrics, Oxford, UK) combined with two force plates (AMTI, Watertown, MA, USA). A $12 \%$ ramp was specifically designed to allow force plates recording during walking. ${ }^{14}$

During a first acquisition, the subject was asked to stand still during $2 \mathrm{~s}$ with his feet parallel. He had to stay as much as possible without any flexion or extension of the lower limb, keep his eyes opened and look straight ahead. Then, three walking conditions at comfortable speed were recorded: on level ground, on a $12 \%\left(7^{\circ}\right)$ ramp ascent and on a $12 \%\left(7^{\circ}\right)$ ramp descent of $6.2 \mathrm{~m}$ long. At least, three clean trials (meaning with the whole foot on the force plate) were processed for every participant with each foot.

\section{Data analysis}

The preliminary static acquisition was used to define the local anatomical coordinate systems associated to each body segment. ${ }^{14}$ The angular position of the joints were then computed as the variation, from the static reference, of the angular position of the proximal segment relatively to the distal one. The joint moments were computed at the joint centers, by an inverse dynamic procedure.

To obtain the ankle moment/angle curve, the evolution of the moment is then drawn as a function of the angle. The Figure 1 shows a typical pattern of this curve. On the graph, the heel strike corresponds to the number 1 . From point 1 to point 2, lies the foot flattening. The number 5 indicates the end of the stance phase.

The following parameters have also been extracted (Figure 1):

- Range of motion (arrow on the Figure 1) computed as the difference between the maximal dorsiflexion (number 4 on Figure 1) and maximal plantarflexion (number 2 on Figure 1),

- Equilibrium point (number 3 on Figure 1) as defined by William et al. corresponding to the angle reached when the moment equals zero during the period between the foot flat and the maximum dorsiflexion. $^{18}$

- Hysteresis (gray area on the Figure 1) defined as the net energy loss of the system, computed on the entire gait cycle as: $\int M d(\theta)$ (with $\mathrm{M}$ the moment and $\theta$ the ankle angle), ${ }^{3}$

- Late stance energy released (black triangle on Figure 1) corresponding to the energy released from the 


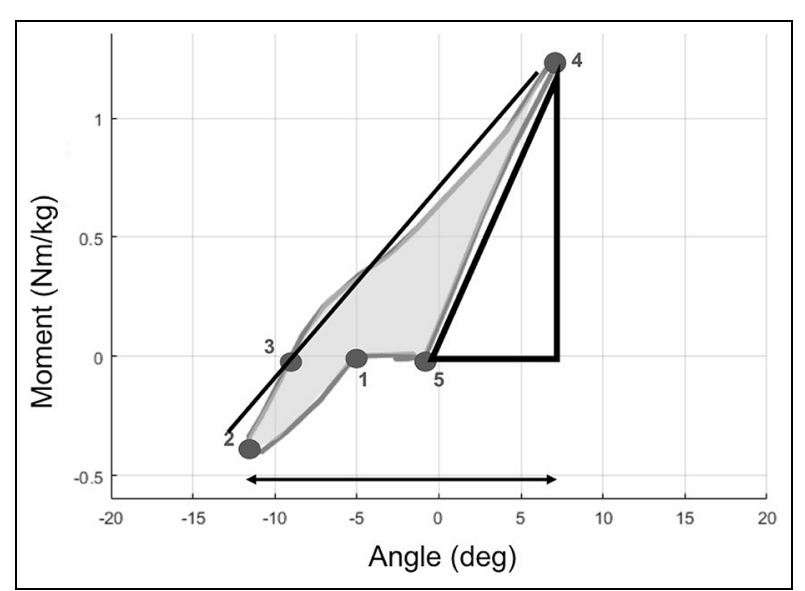

Figure I. Ankle moment $(\mathrm{Nm} / \mathrm{kg}$ ) versus ankle angle (degrees) on level ground (dorsiflexion is positive and plantarflexion is negative)-Number I: heel strike, Number 2: maximal plantarflexion-Number 3: equilibrium point-Number 4: maximal dorsiflexion-Number 5: toe off, the black triangle area correspond to the late stance energy release, the gray area to the hysteresis.

maximal dorsiflexion of the ankle to the end of the stance phase (toe off - number 5 on Figure 1).

- Quasi-stiffness (black line on the Figure 1) computed as the slope of the line that best fits the MAC of the ankle during the unipodal stance phase.

- Global range of motion computed as the range between maximal dorsiflexion reached during slope ascent and the maximal plantarflexion reached during slope descent.

\section{Statistics}

The differences of the parameters observed between level and the other conditions were compared using the analysis of variance (ANOVA) with "ankle type" (ESR, Elan $^{\mathrm{TM}}$, Propriofoot ${ }^{\mathrm{TM}}$, Meridium $^{\mathrm{TM}}$ ) as factors. Post hoc analyses were conducted using Tukey tests. The level of significance was set at $p<0.05$. In the results part, only significant differences will be reported.

\section{Results}

Table 3 summarizes the values of the different parameters per condition averaged over all the participants whatever the foot used.

The following results refer only to the prosthetic side, not to the sound side of the participants.

The effect of the foot worn by the participant has been studied in the three conditions: level ground, ascent, descent. The speed was not statistically different for each participant whatever the foot for the three situations.

Figure 2 shows the typical patterns of the momentangle curves. They were similar among the participants while they were wearing the same foot except for the Elan $^{\mathrm{TM}}$ for which two profiles could be observed (Figure 2(b) and (c)).

\section{Range of motion (ROM)}

The highest plantarflexion of the prosthetic ankle complex among all prosthetic feet was observed when participants walked down the ramp when using the Meridium $^{\mathrm{TM}}$ foot and could reached $17^{\circ}$ (Table 3 ).

The maximal dorsiflexion was also observed with the Meridium ${ }^{\mathrm{TM}}\left(10^{\circ}\right)$ in descent. On the contrary, the Elan $^{\mathrm{TM}}$ presented the lowest value of maximal dorsiflexion in ascent $\left(9^{\circ}\right)$ and maximal plantarflexion in descent $\left(12^{\circ}\right)$. The difference of the maximal dorsiflexion was

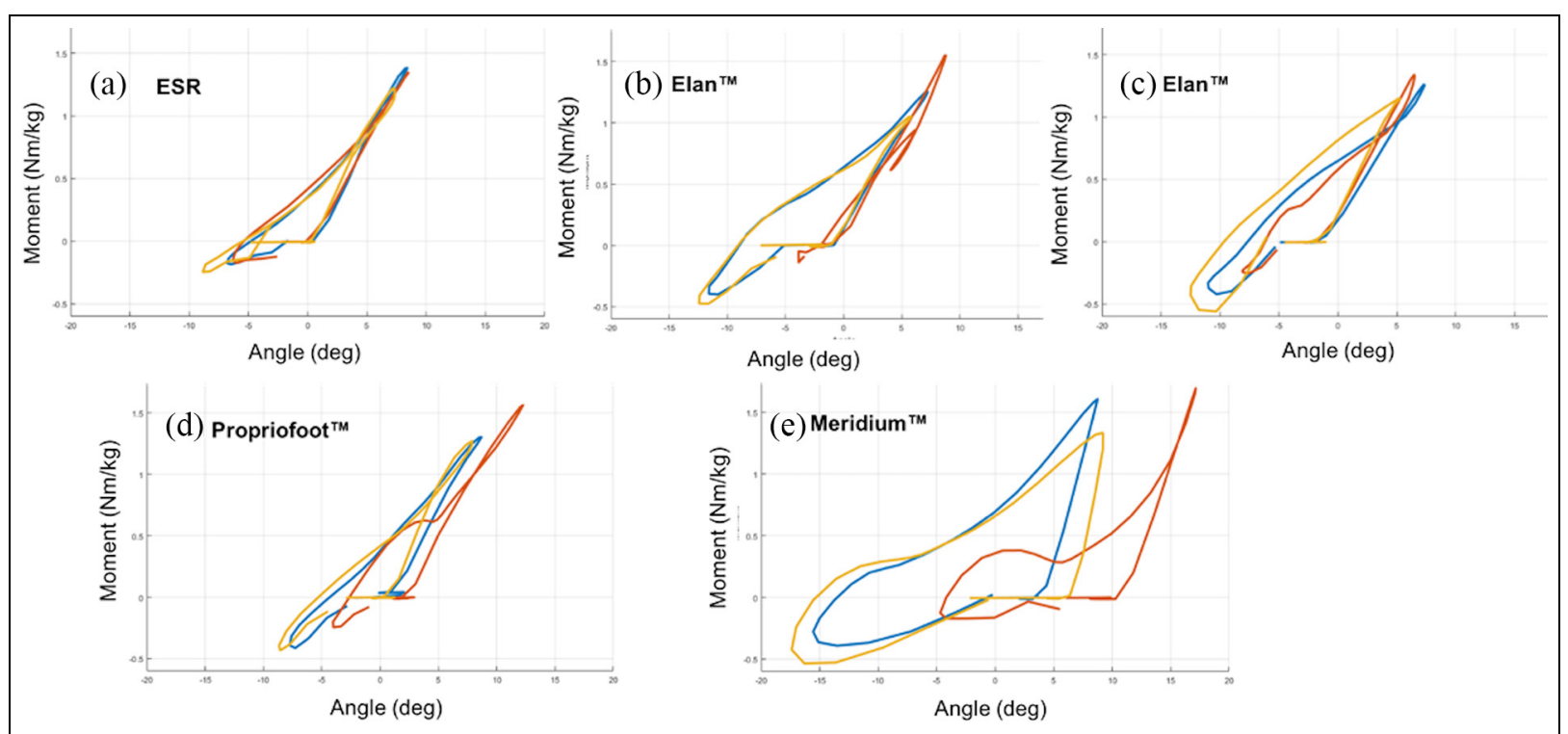

Figure 2. Typical patterns of the ankle moment $(\mathrm{Nm} / \mathrm{kg})$ versus ankle angle (degrees) in three different conditions (ascent $=$ red, level = blue, descent = yellow) for the different feet tested-each subfigure corresponds to the average of one participant describing the typical patterns obtained (one for each foot except the Elan which had two different patterns (b and c).[AQ: I]. 
Table 3. Mean values (standard deviation) for each foot of all participants for each parameter and the three different conditions.

\begin{tabular}{|c|c|c|c|c|}
\hline Variable & Foot & Down & Level & Up \\
\hline \multirow[t]{4}{*}{ Speed $(\mathrm{m} / \mathrm{s})$} & ESR & I.3 (0.2) & $1.2(0.2)$ & $1.2(0.2)$ \\
\hline & Elan & $1.3(0.1)$ & $\mathrm{I} .3(0.2)$ & $\mathrm{I} .3(0.2)$ \\
\hline & Propriofoot & $1.3(0.1)$ & $1.2(0.1)$ & $1.3(0.2)$ \\
\hline & Meridium & I.3 (0.2) & I.3 (0.2) & I.3 (0.2) \\
\hline \multirow[t]{4}{*}{$\operatorname{Max} \mathrm{DF}\left({ }^{\circ}\right)$} & ESR & $7.9(1.6)$ & $9.2(1.8)$ & $10.4(3.0)$ \\
\hline & Elan & $7(0.7)$ & $8.3(0.7)$ & $9(0.2)$ \\
\hline & Propriofoot & $9.6(2.2)$ & $10.5(2.2)$ & $13.5(1.1)$ \\
\hline & Meridium & $9.9(3.9)$ & $9(2.5)$ & $15(3.3)$ \\
\hline \multirow[t]{4}{*}{$\operatorname{Max} \operatorname{PF}\left({ }^{\circ}\right)$} & ESR & $11.4(1.6)$ & $9.6(1.4)$ & $7.1(0.6)$ \\
\hline & Elan & $11.9(0.5)$ & $10.6(0.6)$ & $4.9(2.7)$ \\
\hline & Propriofoot & $\mathrm{II}(\mathrm{I} .5)$ & $10.3(2.0)$ & $4.6(0.7)$ \\
\hline & Meridium & 12.7 (2.7) & II.3 (2.I) & $4.5(1.0)$ \\
\hline \multirow[t]{4}{*}{ Range of motion $\left(^{\circ}\right)$} & ESR & 19.3 & 18.8 & 17.5 \\
\hline & Elan & 18.9 & 18.9 & 13.9 \\
\hline & Propriofoot & 20.6 & 20.8 & I8.1 \\
\hline & Meridium & 22.6 & 20.3 & 19.5 \\
\hline \multirow[t]{4}{*}{ Equilibrium point $\left({ }^{\circ}\right)$} & ESR & $7(1.9)$ & $6.2(1.2)$ & $5.4(0.8)$ \\
\hline & Elan & $5(1.3)$ & 7.1 (1.9) & $2.5(2.8)$ \\
\hline & Propriofoot & $6.2(1.0)$ & $6.6(1.8)$ & $3(0.0)$ \\
\hline & Meridium & I I.2 (2.) & $9.6(2.2)$ & $4.8(1.2)$ \\
\hline \multirow[t]{4}{*}{ Hysteresis $(\mathrm{J} / \mathrm{kg})$} & ESR & $4.2(1.7)$ & $3.7(1.4)$ & $3.0(1.8)$ \\
\hline & Elan & $5.2(2.2)$ & $5.4(1.9)$ & $1.5(1.5)$ \\
\hline & Propriofoot & $3.3(0.6)$ & $3.8(\mathrm{I} .6)$ & 4.1 (I.8) \\
\hline & Meridium & I5.2(2.8) & $10.4(2.4)$ & 7.1 (I.7) \\
\hline \multirow[t]{4}{*}{ Energy propulsion $(\mathrm{J} / \mathrm{kg})$} & ESR & $5.6(1.5)$ & $7.3(3.1)$ & $9.1(4.2)$ \\
\hline & Elan & $4.0(0.3)$ & $5.4(0.4)$ & $6.1(0.8)$ \\
\hline & Propriofoot & $5.6(1.3)$ & $6.7(0.8)$ & $8.0(0.9)$ \\
\hline & Meridium & $2.2(0.5)$ & $3.7(0.9)$ & 4.0 (I.I) \\
\hline \multirow[t]{4}{*}{ Quasi-stiffness $\left(\mathrm{Nm} / \mathrm{kg} /{ }^{\circ}\right)$} & ESR & $0.08(0.01)$ & $0.09(0.01)$ & $0.09(0.01)$ \\
\hline & Elan & $0.08(0.01)$ & $0.1 I(0,00)$ & $0.09(0.02)$ \\
\hline & Propriofoot & $0.07(0.02)$ & $0.08(0.02)$ & $0.08(0.02$ \\
\hline & Meridium & $0.08(0.01)$ & $0.09(0.01)$ & $0.10(0.01)$ \\
\hline \multirow{4}{*}{$\begin{array}{l}\text { Range of motion global including } \\
\text { the three walking conditions }\left(^{\circ}\right)\end{array}$} & ESR & $22(3.3)$ & & \\
\hline & Elan & $20.5(0.9)$ & & \\
\hline & Propriofoot & 24 (1.9) & & \\
\hline & Meridium & $27.8(4.1)$ & & \\
\hline
\end{tabular}

statistically significant for the Meridium ${ }^{\mathrm{TM}}$ compared to the $\operatorname{Elan}^{\mathrm{TM}}(p=0.008)$ and to the $\operatorname{ESR}(p=0.0027)$.

In every situation, the highest ROM was observed with the Meridium ${ }^{\mathrm{TM}}$ (on average, $19.5^{\circ}$ in descent, $20.5^{\circ}$ on level ground, $22.6^{\circ}$ in ascent) and the lowest ROM with the Elan ${ }^{\mathrm{TM}}$ (on average, $18.9^{\circ}$ in descent, $18.9^{\circ}$ on level ground and $13.9^{\circ}$ in ascent).

When considering all the conditions, the global range of motion (from the maximal plantarflexion to the maximal dorsiflexion across all conditions) was also quantified. The highest global range of motion was observed for the Meridium ${ }^{\mathrm{TM}}\left(27.8^{\circ}\right.$ in average) and the lowest for the $\operatorname{Elan}^{\mathrm{TM}}\left(20.5^{\circ}\right)$. The global range of motion was statistically different between the Meridium $^{\mathrm{TM}}$ and the $\operatorname{Elan}^{\mathrm{TM}}(p=0.0358)$.

\section{Equilibrium point}

For the ESR, the equilibrium point was similar in the three conditions (no shift of the curve along the $\mathrm{X}$ axis) (Figure 2(a)). For the Elan ${ }^{\mathrm{TM}}$, the equilibrium point was not shifted for the first characteristic pattern
(Figure 2(b)). This was not true for the second characteristic pattern (Figure 2(c)). For the Propriofoot ${ }^{\mathrm{TM}}$, there was no shift of the equilibrium point between level ground and descent. On the contrary, a shift could be observed between level ground and ascent (Figure 2(d)). Finally, there is a shift of the equilibrium point with the Meridium ${ }^{\mathrm{TM}}$, between level ground and descent as between level ground and ascent (Figure 1(e)).

\section{Hysteresis}

The hysteresis (Figure 2 and Table 3) was different for the 4 feet and the three conditions. The Propriofoot ${ }^{\mathrm{TM}}$ and the ESR presented the lowest hysteresis and their averaged values remain similar in all conditions. The Meridium $^{\text {TM }}$ hysteresis was statistically higher (2-3 times) than the one of the other 3 feet $(p=0.001)$.

Concerning the Elan $^{\mathrm{TM}}, 2$ different behaviors were observed:

- For two subjects, during the ascent, the hysteresis was almost null: all the energy stored was returned 
(Figure 2(b)). On level ground and in descent, the hysteresis was not null but was similar in both conditions.

- For two subjects, on the contrary, the MAC showed a real loading phase (Figure 2(c)) and the hysteresis changed depending on the condition $\left(\right.$ Area $_{\text {descent }}>$ Area $_{\text {flat }}>$ Area $_{\text {ascent }}$ ).

\section{Late stance energy released}

Concerning the late stance energy released corresponding to the propulsion energy, the same trends as for the hysteresis could be observed for all feet depending on the situation. The energy released was the lowest in descent and the highest in ascent. On level ground, it was higher than in descent and lower than in ascent. The foot with the lowest energy for the propulsion was the Meridium $^{\mathrm{TM}}$.

\section{Quasi-stiffness}

The values of stiffness quantified for the different feet and conditions did not reveal statistical differences.

\section{Discussion}

The first aim of the study was to investigate the ability of different parameters extracted from the momentangle curve in the quantification of prosthetic feet adaptation between level and slope locomotion. To this purpose, some parameters coming from the literature were quantified from gait analysis data of five people with transtibial amputation successively fitted with 3 MPAs compared to their usual ESR foot in three walking conditions (level ground, upslope, and downslope).

The present study confirms the interest of the MAC for the design and the evaluation of biomimetic prosthetic feet as already suggested in the literature. ${ }^{3,15}$ The results also support that parameters extracted from this curve are very useful to quantify the adaptation of MPA. ${ }^{18}$ Indeed, these parameters are synthetic of the role of the ankle-foot complex, which allow the comparison of different technological design. ${ }^{19}$

The Propriofoot ${ }^{\mathrm{TM}}$ was the first MPA. The electric actuator is able to modify the ankle position during the swing phase toward dorsiflexion during slope ascent and toward plantarflexion during slope descent. It results in a shift of the ankle MAC on the angle axis that could be observed between the different conditions. This adaptation is higher during slope ascent than during slope descent. The global pattern of the curve remains the same, showing that the behavior of the foot is not modified during the stance phase. ${ }^{7}$ This is consistent with the design of the foot as the observed range of motion is due to the foot structure deformation under loading (like ESR feet). The results also show that the ankle angle adaptation allows to decrease the energy return during slope descent and to increase it during slope ascent in an order of magnitude close to the value obtained with ESR feet.

The Elan ${ }^{\mathrm{TM}}$ foot adapts to varying terrain by modifying the damping coefficient of the ankle unit accounting for $9^{\circ}$ of flexion extension. ${ }^{9,20}$ The ankle MAC is not shifted on the angle axis but the hysteresis is adapted to the slope to dissipate more energy during slope descent or less during slope ascent. The increase of the range of motion in slope, consecutive to the increase of plantarflexion in descent and dorsiflexion in ascent is limited compared to the other feet tested in this study. Finally, two profiles of MAC could be observed, corresponding to a variable adaptation of the foot depending on the individual setting. In particular, the setting of the hydraulic valve can impact the possible range of motion of the ankle.

The behavior of the Meridium ${ }^{\mathrm{TM}}$ is also adapted to varying terrain by modifying the hydraulic ankle behavior but on a wider range of motion than the Elan ${ }^{\mathrm{TM}}$ and is able to hydraulically lock the ankle at a given angle. $^{10,21}$ The ankle MAC was shifted along the angle axis toward dorsiflexion during slope ascent until 15 degrees of maximal dorsiflexion and toward plantarflexion during slope descent until 12.7 degrees. These amplitudes allowed the highest global range of motion observed in the study giving a wide ability to adapt to the different walking conditions. The hysteresis of the curve during stance is also modified showing an increase of the dissipated energy during slope descent and a decrease of the dissipated energy during slope ascent. However, the returned energy during propulsion is the smallest among the feet tested in this study. The Meridium is the foot that presents the highest differences of hysteresis of the MAC between the different walking conditions. During the descent, more energy is lost compared to the other tested feet, which helps the subject in controlling his descent. In ascent, the hysteresis is decreased compared to level ground and descent. Thus, the dissipation of energy is limited in this condition where more energy is needed to go up the slope. However, it results in a low available power for the propulsion compared to the other feet. This trend is consistent with the functioning of the foot as the net external energy loss is directly linked to the energy that can be returned and used for propulsion.

Suggestive feedbacks and clinical functional test have been investigated in the companion article published in 2019 by Thomas-Pohl et al. ${ }^{22}$ Basically, the participants found the MPAs heavier and less reactive for the propulsion.

\section{Study limitations}

The first limitation of the present study is the sample size which limits the power of the statistical analysis. Also, the population recruited was young (four of the participants were younger than 35 years old) and very active (military population).

The definition of the ankle center for the prosthetic feet can be also discussed. Indeed, for carbon-leaf foot 
design, there is no mechanical axis and the position of an ankle center is questionable. ${ }^{23}$ On the contrary, for the 3 MPAs tested in the study, the mechanical axis could be visually identified, which ensures a consistent comparison between the feet.

The marker placement between sessions could introduced biases on the computation of the joint angles and moments, even if it was limited by the protocol.

Finally, even with an adaptation time and a fitting performed always by the same expert prosthetist, different profiles with the use of the Elan can be observed that can be related to the individual needs of the patient that the prosthetist must have to fulfill.

\section{Conclusion}

The results of the present study show that the parameters that can be extracted from the moment/angle curve of prosthetic ankle foot are useful for the evaluation of their behavior in situ as it allowed to differentiate the different MPA. The main advantage of this curve is to synthetize the main parameters that have an impact on the gait and that are in relation with the design of the foot.

This independent study has allowed to compare microprocessor-controlled ankle with ESR foot by providing meaningful parameters in different walking conditions. This quantitative evaluation shows that every MPA adapts their range of motion to the condition but that these adaptations result in a modification of the available energy for the propulsion, which must be taken into account according to the activity of the person.

Thus, it confirms that the choice of the more adapted prosthetic foot to a given person is a trade-off between comfort/propulsion and project life/compensations. The present study will be completed by the investigation of the overlying segments adaptation to characterize immediate compensations that can be observed when wearing different prosthetic feet.

\section{Declaration of conflicting interests}

The author(s) declared no potential conflicts of interest with respect to the research, authorship, and/or publication of this article.

\section{Funding}

The author(s) received no financial support for the research, authorship, and/or publication of this article.

\section{ORCID iD}

Julie Davot (iD https://orcid.org/0000-0001-6663-5840

\section{References}

1. Jarvis HL, Bennett AN, Twiste M, et al. Temporal spatial and metabolic measures of walking in highly functional individuals with lower limb amputations. Arch Phys Med Rehabil 2017; 98(7): 1389-1399.

2. Esposito ER, Rodriguez KM, Ràbago CA, et al. Does unilateral transtibial amputation lead to greater metabolic demand during walking. J Rehabil Res Dev 2014; 51(8): 1287-1296.

3. Hansen AH, Childress DS, Miff SC, et al. The human ankle during walking: implications for design of biomimetic ankle prostheses. J Biomech 2004; 37(10): $1467-$ 1474.

4. Vickers DR, Palk C, McIntosh AS, et al. Elderly unilateral transtibial amputee gait on an inclined walkway: a biomechanical analysis. Gait Posture 2008; 27(3): 518-529.

5. Vrieling AH, van Keeken HG, Schoppen T, et al. Uphill and downhill walking in unilateral lower limb amputees. Gait Posture 2008; 28(2): 235-242.

6. Langlois K, Villa C, Xavier B, et al. Influence of physical capacities of males with transtibial amputation on gait adjustments on sloped surfaces. J Rehabil Res Dev 2014; 51(2): 193-200.

7. Fradet L, Alimusaj M, Braatz F, et al. Biomechanical analysis of ramp ambulation of transtibial amputees with an adaptive ankle foot system. Gait Posture 2010; 32(2): 191-198.

8. Delussu AS, Brunelli S, Paradisi F, et al. Assessment of the effects of carbon fiber and bionic foot during overground and treadmill walking in transtibial amputees. Gait Posture 2013; 38(4): 876-882.

9. Struchkov V and Buckley JG. Biomechanics of ramp descent in unilateral trans-tibial amputees: comparison of a microprocessor controlled foot with conventional ankle-foot mechanisms. Clin Biomech 2016; 32: 164-170.

10. Ernst M, Altenburg B, Bellmann M, et al. Standing on slopes-how current microprocessor-controlled prosthetic feet support transtibial and transfemoral amputees in an everyday task. $J$ Neuroeng Rehabil 2017; 14(1): $1-16$.

11. Ko CY, Kim SB, Kim JK, et al. Comparison of ankle angle adaptations of prosthetic feet with and without adaptive ankle angle during level ground, ramp, and stair ambulations of a transtibial amputee: a pilot study. Int $J$ Precis Eng Manuf 2014; 15(12): 2689-2693.

12. Schmalz T, Altenburg B, Ernst M, et al. Ramp walking with abruptly changing inclines: motion pattern of TT amputees fitted with a microprocessor-controlled and a conventional prosthetic foot. Gait Posture. 2018; 65.[AQ: 2]

13. Hansen AH, Miff SC, Childress DS, et al. Net external energy of the biologic and prosthetic ankle during gait initiation. Gait Posture 2010; 31(1): 13-17.

14. Pillet $\mathrm{H}$, Drevelle $\mathrm{X}$, Bonnet $\mathrm{X}$, et al. APSIC: training and fitting amputees during situations of daily living. Irbm 2014; 35(2): 60-65.

15. Frossard L, Leech B and Pitkin M. Automated characterization of ankle stiffness of prosthetic feet fitted to bone-anchored transtibial prosthesis. IEEE Trans Biomed Eng. Epub ahead of print 13 March 2019. DOI: 10.1109/ TBME.2019.2904713.

16. Shamaei K, Sawicki GS and Dollar AM. Estimation of quasi-stiffness and propulsive work of the human ankle in the stance phase of walking. PLoS One 2013; 8(3): e59935. 
17. Hansen A and Starker F. Prosthetic foot principles and their influence on gait. Case Rep Pediatr 2015; 2015: 406359.

18. Williams RJ, Hansen AH and Gard SA. Prosthetic anklefoot mechanism capable of automatic adaptation to the walking surface. J Biomech Eng 2009; 131(3): 035002.

19. Versluys R, Beyl P, Van Damme M, et al. Prosthetic feet: state-of-the-art review and the importance of mimicking human anklefoot biomechanics. Disabil Rehabil Assist Technol 2009; 4(2): 65-75.

20. Koehler-McNicholas SR, Nickel EA, Medvec J, et al. The influence of a hydraulic prosthetic ankle on residual limb loading during sloped walking. PLoS One 2017; 12(3): $1-18$.
21. Schmalz T, Altenburg B, Ernst M, et al. Lower limb amputee gait characteristics on a specifically designed test ramp: preliminary results of a biomechanical comparison of two prosthetic foot concepts. Gait Posture 2019; 68: 161-167.

22. Thomas-Pohl M, Villa C, Davot J, et al. Microprocessor prosthetic ankles: comparative biomechanical evaluation of people with transtibial traumatic amputation during standing on level ground and slope. Disabil Rehabil Assist Technol 2021; 16(1): 17-26.

23. Prince F, Winter DA, Sjonnesen G, et al. A new technique for the calculation of the energy stored, dissipated, and recovered in different ankle-foot prostheses. IEEE Trans Rehabil Eng 1994; 2(4): 247-255. 